\title{
Investigation of droplets released during digestive endoscopy using a high-speed camera (with video): a pilot study
}

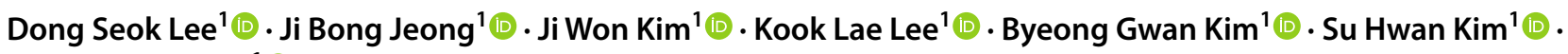 \\ Hyoun Woo Kang ${ }^{1}$ (D)
}

Received: 1 February 2021 / Accepted: 2 June 2021 / Published online: 11 June 2021

(c) The Author(s), under exclusive licence to Springer Science+Business Media, LLC, part of Springer Nature 2021

\begin{abstract}
Background A large release of droplets is often expected around the periphery of the digestive endoscope insertion site. Therefore, a sense of alarm over infection because of droplets that may be released during digestive endoscopy examination is increasing. This study aimed to investigate the droplets released during digestive endoscopy using a high-speed camera. Methods We utilized a high-speed camera (FASTCAM SA-3, Photron Limited) capable of recording small, transparent droplets with a black background and high-brightness lighting. The obtained video files were analyzed using post-processing software. We divided the 20 models into the control (a spray bottle model and a cough model) and experimental groups (digestive endoscopy models). The sedative, proficiency of digestive endoscopy and the amount of gas injected were modulated to change the level of released droplets.

Results For the control groups, droplets were clearly observed using a high-speed camera. However, no droplet larger than $10 \mu \mathrm{m}$ in size was observed in the experimental groups. Furthermore, the changes in the sedative, proficiency of digestive endoscopy, and amount of gas injected did not affect droplet formation.

Conclusions Based on high-speed camera photography, the risk of droplet generation during digestive endoscopy was not higher than that during violent expiratory events, such as coughing and sneezing.
\end{abstract}

Keywords Droplets · Endoscopy $\cdot$ COVID-19 $\cdot$ Coronavirus $\cdot$ High-speed camera

The coronavirus disease (COVID-19) outbreak, which began in November 2019, has rapidly spread worldwide [1]. Droplets released from the mouth of a COVID-19-infected patient are an important source of infection. Droplets released from an infected patient's sneeze are capable of traveling a distance of over $2 \mathrm{~m}$ [2-4].

It is thought that a large release of droplets is expected around the periphery of the digestive endoscope insertion site. Therefore, a sense of alarm over infection through droplets that may be released during digestive endoscopy examination is increasing [5]. The epidemic prevention guidelines

Dong Seok Lee and Ji Bong Jeong contributed equally to this work as first authors.

\footnotetext{
Ji Won Kim

kjwjor@snu.ac.kr

1 Department of Gastroenterology, Seoul National University Boramae Medical Center, Seoul National University College of Medicine, Seoul, Korea
}

for digestive endoscopy against COVID-19 are set around a standard of the 2-m travel distance of droplets released from a normal sneezing patient and have no clear standard of the travel distance of droplets [6]. Therefore, most medical teams working in the digestive endoscopy room wear N95 masks and face shields, goggles, level-3 protective suits, and gloves, while minimizing the number of endoscopies performed. However, because of the excessive wearing of protective suiting, the accuracy of endoscopies is decreasing, while the procedure-related fatigue levels are increasing. Furthermore, as the duration of the procedure increases, the number of endoscopies performed decreases, which has a negative impact on patients.

Therefore, the aim of this study was to analyze the travel distance and range of droplets released during digestive endoscopy using a high-speed camera to aid in the development of epidemic prevention guidelines related to digestive endoscopy. 


\section{Materials and methods}

\section{Study population and ethical considerations}

From May 2020 to June 2020 we investigated droplets from 20 models. The patients enrolled in the study and partaking in digestive endoscopy had no severe acute respiratory syndrome coronavirus 2 (SARS-CoV-2) infection at 14 days prior to digestive endoscopy. Their age ranged from 21 to 75 years, with no history of traveling to or residing in countries at risk. The exclusion criteria were as follows: respiratory symptoms (coughing and difficulty breathing), fever, history of traveling to or residing in countries at risk without reporting SARS-CoV-2 infection at 14 days prior to the examination, swallowing impediments, the presence of aspiration pneumonia, and inability to undergo gastrointestinal endoscopic examinations. Regarding the droplet generated during digestive endoscopy, as our aim was to analyze only the droplets released through the gastrointestinal tract, patients with respiratory disease who could release sputum did not participate. This study was approved by the Boramae Hospital Institutional Review Board (IRB) (IRB number 07-202017). Informed consent was obtained from the patients.

Five spray bottle models, five normal cough models, and 10 digestive endoscopy examinees were analyzed in this study. The following subcases were constructed with 20 models: case A [spray bottles $(n=5)$ ]; case $\mathrm{B}$ [cough models $(n=5)$ ]; cases $\mathrm{C}, \mathrm{D}$, and $\mathrm{E}$ [gastroscopy (EGD) models $(\mathrm{n}=5)$ ]; and cases F, G, and H [colonoscopy (CFS) models $(\mathrm{n}=5)]$ (Fig. 1).

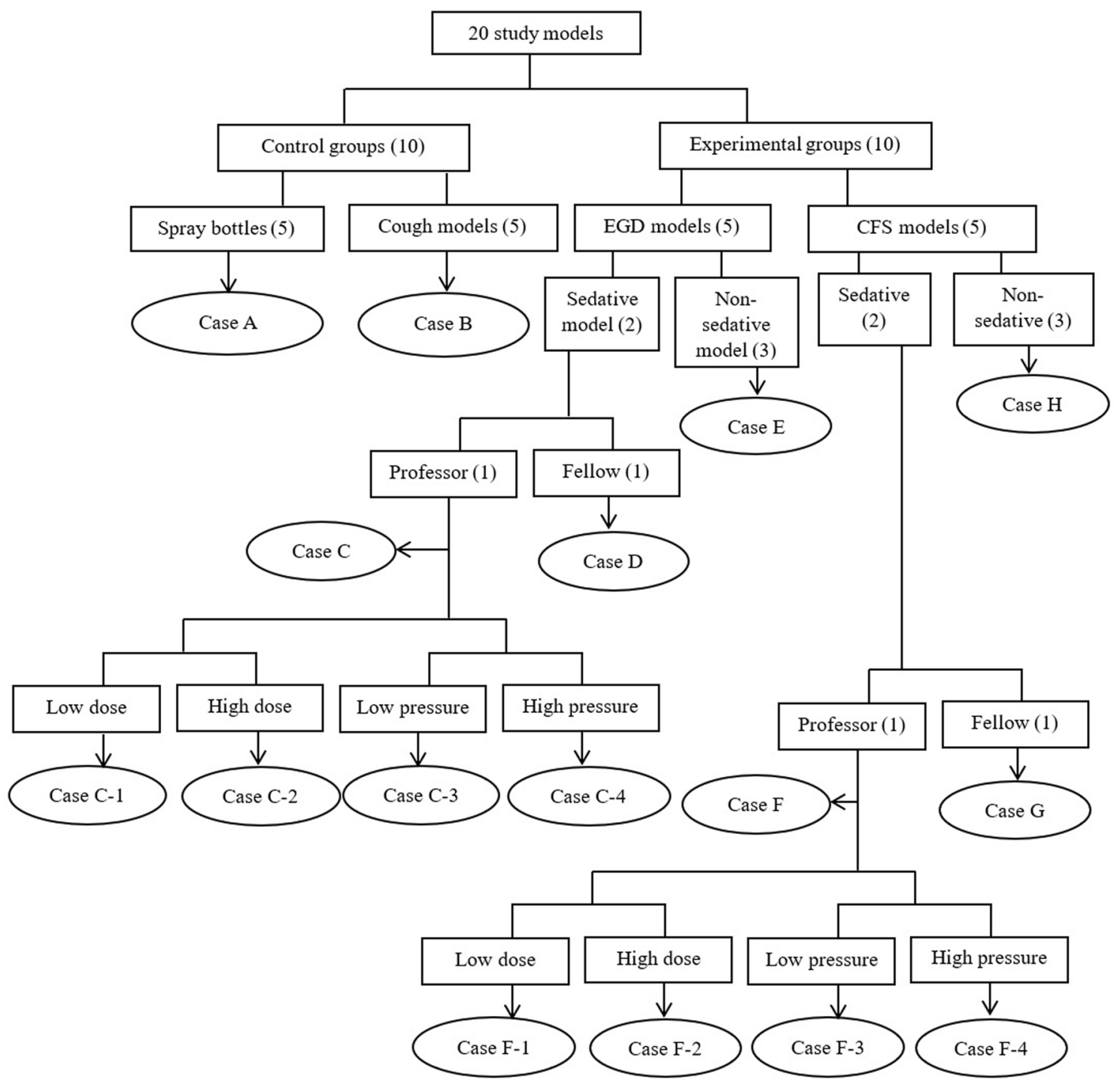

Fig. 1 Distribution of different types of models. EGD, gastroscopy; CFS, colonoscopy 


\section{Details of the measurement of the droplets}

The high-speed camera's photographs show in slow motion instantaneous phenomena, which cannot be photographed with a normal video or film camera, to aid in thoroughly confirming and understanding rapid forms with the naked eye. The movement of an arrow and the migration of a missile can be photographed, and even the evaporation of water and movement of precision explosives can be saved. As the droplets generated during digestive endoscopy are small, transparent, and move at a rapid pace, they are difficult to be confirmed with a naked eye or with normal cameras. Thus, we used a high-speed camera (FASTCAM SA-Z; Photron Limited, Tokyo, Japan), which can photograph 1000 frames/s, and it was calibrated to distinguish particle sizes of $20 \mu \mathrm{m}$ (Fig. 2a). The measurement targets were the size of the droplet, travel distance, emission angle, and emission speed. For droplet determination, the examinee's oral cavity and anal periphery were filmed.

To film transparent droplets, a black background and high-brightness lighting were used in filming (Fig. 2b). To confirm the existence of the droplet on the high-speed camera, a coughing patient model in the state of not wearing the mouthpiece and the spray bottle model were used as the control groups. These groups (spray bottles and cough models) were compared with the droplet release photography of the experimental groups (digestive endoscopy models). Five patients undergoing EGD and five patients undergoing CSF participated in the control groups for the droplet release examination. To minimize stress of the participants,
Fig. 2 Experimental set-up for visualization of the droplets. a The findings using a high-speed camera (FASTCAM SA-3, Photron Limited, Tokyo, Japan) with a black background and high-brightness lighting and $\mathbf{b}$ post-processing of the events using the high-speed camera are presented
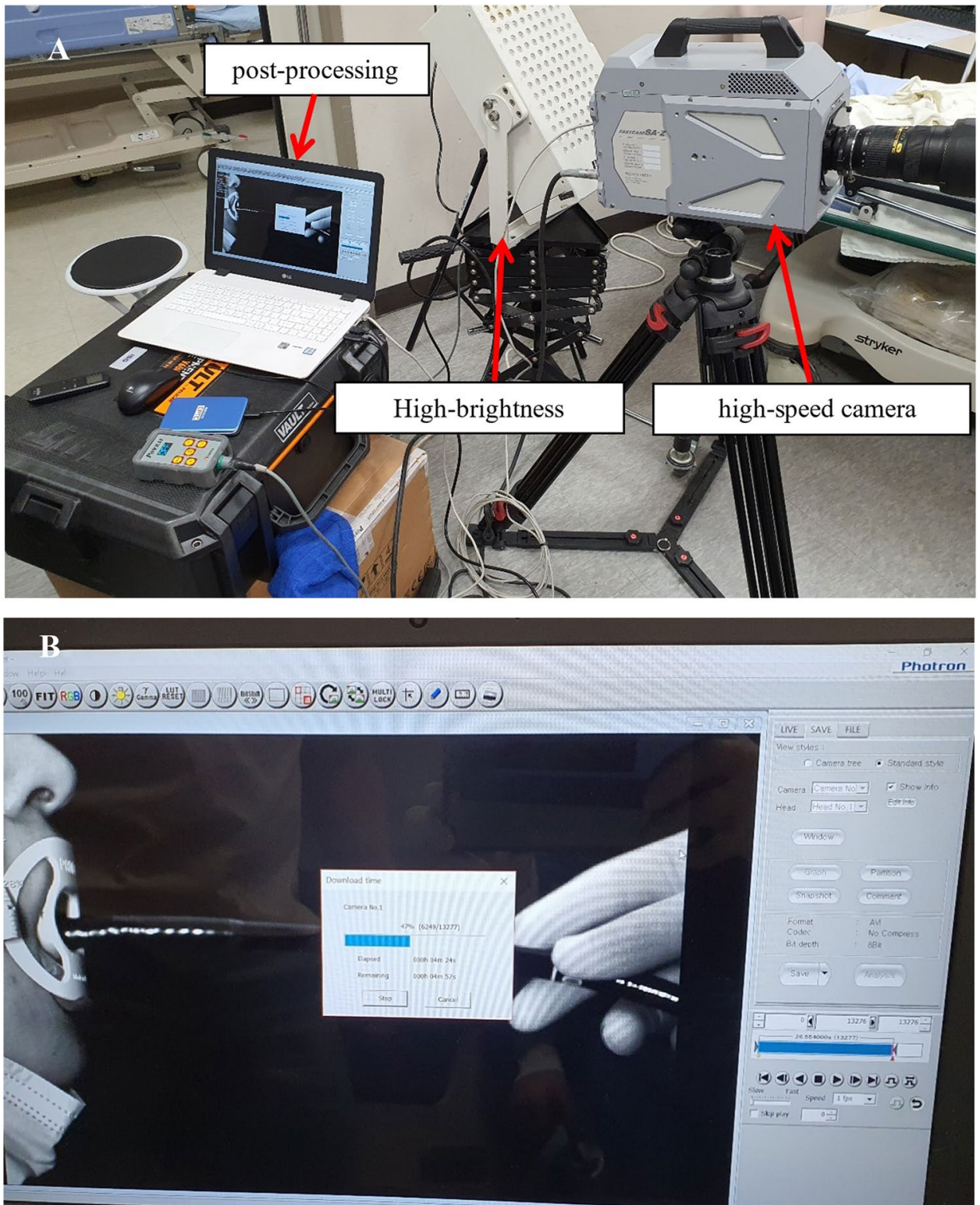
blindfolds were used. The high-speed film footage was transferred to a computer, and the images were processed before analyzing the existence of droplets, which had an average duration of $1 \mathrm{~h}$. Droplet generation was adjusted based on the use of sedatives, endoscopy procedure experience, and the amount of gas injected, and the analysis was performed, focusing on the insertion and removal points of the endoscope, which was expected to produce the most droplet generation.

\section{Results}

In the control groups (case A) of the spray bottle and cough models (case B), droplet particles were clearly observed. In the spray bottle model, the moving droplets were relatively uniform in size and direction. The droplet spread out within the radiation angle range of approximately 60 degrees, and the diameter of the droplet was 200-1000 $\mu \mathrm{m}$. Droplets moving according to the movement of the tongue and lips were observed in the cough model. In the cough model, the droplets spread within the radial angle range of approximately 105 degrees, and the diameter of the droplets ranged from 50 to $1000 \mu \mathrm{m}$. However, in the EGD and CSF examinations, regardless of the use of sedatives, endoscopy proficiency, and amount of gas injected, no droplets moved parallel to the plane of the endoscope around the endoscope insertion site periphery (Fig. 3a-c). During EGD, saliva flowed down the sides of the mouth, and in CSF, intestinal fluids flowed down around the anus. Figure 1 and Video 1 demonstrates droplets from three representative models.

Fig. 3 Visualization of a droplet recorded at 1,000 FPS. a Large droplets released in the spray bottle model. b Droplets from a cough in the normal coughing model. c The images obtained around the periphery of the EGD. FPS, frame per second; EGD, gastroscopy
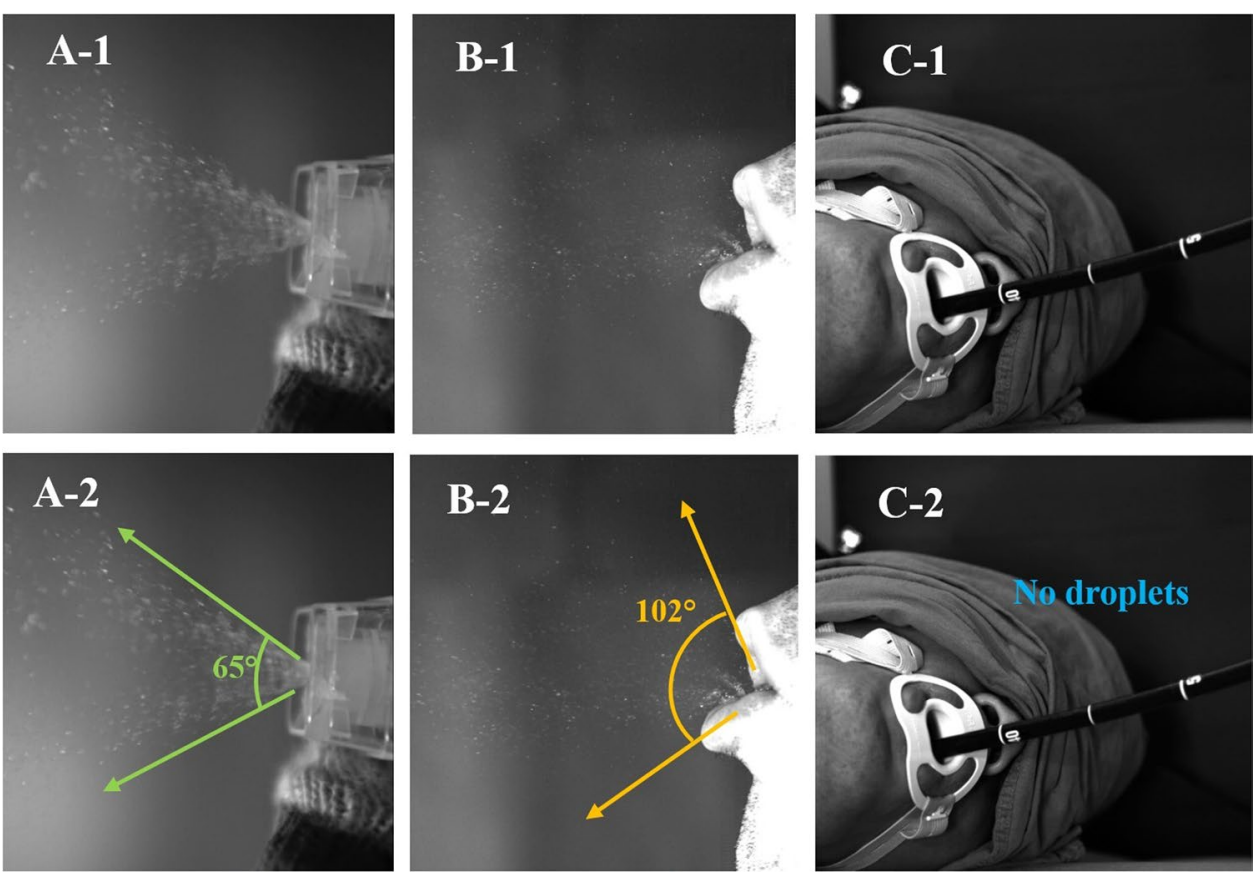
This study had some limitations. First, much time was spent on the high-speed camera post-processing procedure and, therefore, the number of study participants was low. Second, COVID-19-infected patients were excluded from the study. Therefore, further large-scale studies are required to overcome these limitations. Third, there was a limitation when measuring the exact particle size because the droplets move under the influence of the particle size, spray direction, gravity, and the flow of the surrounding atmosphere. In the case of the spray bottle model, the size of the nozzle is different for each manufacturer. Moreover, in the case of the cough model, the amount and size of droplets released may vary depending on the patient. In addition, as the movement direction of the particles may be different depending on the air circulation and gravity in the measurement room, it is necessary to record from three directions to measure the movement direction of the droplet. In a future research, we will consider shooting with special dyes for shooting to make accurate particle size measurements.

In conclusion, based on high-speed camera photography, no droplets were observed during digestive endoscopy. The mouthpiece and anus play a role in blocking the release of droplets at the entry site of the endoscope. We believe that the risk of droplet generation during digestive endoscopy was not higher than that of violent expiratory events, such as coughing and sneezing. This study's findings can be effective for the development of preventative guidelines against COVID-19 in digestive endoscopy.

Supplementary Information The online version contains supplementary material available at https://doi.org/10.1007/s00464-021-08581-0.

Acknowledgements The authors appreciate the technical support provided by the Institute of Construction and Environmental Engineering at Seoul National University.

Author contributions Concept-DSL, JBJ, and JWK; Design-DSL, JBJ, and JWK; Supervision-KLL and JWK; Resources-DSL, JBJ, and BGK; Materials-DSL and JBJ; Data collection and/or processing-DSL, JWK, JBJ, and BGK; Analysis and/or interpretation-DSL and JWK; Literature search-DSL, SHK, and HWK; Writing manuscript-DSL and JBJ; Critical reviews-KLL and JWK.
Funding None.

\section{Declarations}

Disclosures Dong Seok Lee, Ji Bong Jeong, Ji Won Kim, Kook Lae Lee, Byeong Gwan Kim, Su Hwan Kim, and Hyoun Woo Kang have no conflicts of interest or financial ties to disclose.

\section{References}

1. Phelan AL, Katz R, Gostin LO (2020) The novel coronavirus originating in Wuhan, China: challenges for global health governance. JAMA 323:709-710

2. Bourouiba L (2016) Images in clinical medicine. A Sneeze. N Engl J Med 375:e15

3. Bourouiba L, Dehandschoewercker E, Bush J (2014) Violent expiratory events: on coughing and sneezing. J Fluid Mech 745:537-563

4. Scharfman B, Techet A, Bush J, Bourouiba L (2016) Visualization of sneeze ejecta: steps of fluid fragmentation leading to respiratory droplets. Exp Fluids 57:24

5. Chiu PWY, Ng SC, Inoue H, Reddy DN, Ling HuE, Cho JY, Ho LK, Hewett DG, Chiu HM, Rerknimitr R, Wang HP, Ho SH, Seo DW, Goh KL, Tajiri H, Kitano S, Chan FKL (2020) Practice of endoscopy during COVID-19 pandemic: position statements of the Asian Pacific society for digestive endoscopy (APSDECOVID statements). Gut 69:991-996

6. Wong TW, Lee CK, Tam W, Lau JT, Yu TS, Lui SF, Chan PK, Li Y, Bresee JS, Sung JJ, Parashar UD, Outbreak Study Group (2004) Cluster of SARS among medical students exposed to single patient, Hong Kong. Emerg Infect Dis 10:269-276

7. Tran K, Cimon K, Severn M, Pessoa-Silva CL, Conly J (2012) Aerosol generating procedures and risk of transmission of acute respiratory infections to healthcare workers: a systematic review. PLoS ONE 7:e35797

8. Duguid JP (1946) The size and the duration of air-carriage of respiratory droplets and droplet-nuclei. Epidemiol Infect 44:471-479

9. Lee DS, Kim JW (2021) Analysis of droplets generated during gastrointestinal endoscopic examinations using a high speed camera. Endoscopy 53:207-208

Publisher's Note Springer Nature remains neutral with regard to jurisdictional claims in published maps and institutional affiliations. 